\title{
Suppression of osteogenic-like differentiation in human renal interstitial fibroblasts by miRNA-410-3p through MSX2
}

\author{
Yu Cui", Feng Zeng", Zewu Zhu, Fang Huang, Jinbo Chen, Cheng He, Yang Li, Zhiyong Chen, \\ Zhongqing Yang, Xiongbing $\mathrm{Zu}$, Hequn Chen \\ Department of Urology, Xiangya Hospital, Central South University, Changsha, China \\ Contributions: (I) Conception and design: Y Cui, H Chen; (II) Administrative support: Y Cui, F Zeng, H Chen; (III) Provision of study materials \\ or patients: Y Cui, Z Zhu, F Huang, J Chen; (IV) Collection and assembly of data: F Zeng, Y Li, Z Chen, Z Yang, C He; (V) Data analysis and \\ interpretation: X Zu, H Chen, F Zeng; (VI) Manuscript writing: All authors; (VII) Final approval of manuscript: All authors. \\ \#These authors contributed equally to this work. \\ Correspondence to: Hequn Chen. Department of Urology, Xiangya Hospital, Central South University, Changsha, China. Email: chenhequnxy@126.com.
}

Background: The aim of this stay was to determine the effect of calcium ions in promoting osteogenic-like differentiation in human renal interstitial fibroblasts (hRIFs). The role of miRNA-410-3p in upregulating Msh homeobox 2 (MSX2) level in hRIFs was also investigated.

Methods: Quantitative polymerase chain reaction (qPCR) analysis was used to assess the expression levels of miRNA-410-3p in Randall's plaque (RP) and normal renal papillary (nRP) tissues. Furthermore, the expression levels of osteogenesis-related protein in the RP and nRP tissues were assessed with qPCR and immunohistochemistry (IHC). hRIFs were cultured from isolated human kidney papilla before treatment with calcium chloride or osteogenic medium, and 3-(4,5-dimethylthiazol-2-yl)-2,5-diphenyltetrazolium bromide (MTT) assay was performed at 1, 5, 9, and 14 days post-treatment. Alizarin red staining was used to estimate the deposits of calcium aggregates. After the overexpression or knockdown of miRNA-410-3p, we evaluated the changes in the osteogenic-like differentiation and osteogenesis-related protein by alizarin red staining and qPCR, respectively. A binding relationship between miRNA-410-3p and MSX2 was established through a dual-luciferase reporter gene assay. Rescue experiments demonstrated that miRNA-410-3p regulated the osteogenic-like differentiation by targeting MSX2.

Results: miRNA-410-3p levels were lower in RP tissue than in control nRP tissues. qPCR and IHC showed that the level of runt-related transcription factor 2 (Runx2), osteocalcin (OCN), and osteopontin (OPN) were higher in RP tissues. The calcium deposition of hRIFs showed a time-dependent trend when treated with osteogenic medium or calcium chloride. The overexpression of miRNA-410-3p downregulated the levels of osteogenesis-related expression and attenuated mineralization. The knockdown of miRNA410-3p yielded the opposite trend. Dual-luciferase reporter gene assay and rescue experiments indicated that miRNA-410-3p could target MSX2, while the overexpression of MSX2 reversed the effects of miRNA-4103 p on osteogenic-like differentiation.

Conclusions: The current findings suggest that calcium ions could promote the osteogenic-like differentiation of hRIFs and miRNA-410-3p regulates hRIFs osteogenic-like differentiation by inhibiting MSX2.

Keywords: Kidney calculi; differentiation; epigenetics; miRNA; fibroblasts

Submitted Feb 22, 2020. Accepted for publication Sep 04, 2020.

doi: $10.21037 /$ tau-20-607

View this article at: http://dx.doi.org/10.21037/tau-20-607 


\section{Introduction}

Kidney stones are the most common disease of the urinary tract, and a reported $10-12 \%$ of people in industrialized countries will have a urinary stone in their lifetime (1). Kidney stones affect 1 in 11 people in the USA, with over 600,000 Americans estimated to suffer from urolithiasis every year (2). Approximately $5-10 \%$ of the Chinese population is expected to be diagnosed with urolithiasis, $25 \%$ of which will require hospitalization (3). The main components of kidney stones are calcium stones (about $80 \%)$ with the majority being the calcium oxalate $(\mathrm{CaOx})$ type. The mechanisms of kidney stone development, or lithogenesis, are not completely understood. The major theory for predicting lithogenesis is the presence of Randall's plaque (RP), which appears to be the precursor of kidney stone development (4). One study found that most $\mathrm{CaOx}$ stones were found to be attached to $\mathrm{RP}$ at the renal papilla (5). However, the pathogenesis of RP remains unclear. Hypercalciuria, and the acidification concentration of urine, have all been implicated in RP formation (6). Among these, the involvement of hypercalciuria deserves special focus.

Recently, investigators have found that ectopic calcification is involved in the formation of RP (7). Many studies have shown that typical bone osteoid protein and hydroxyapatite mineralization are found in RP (8-10). He et al. discovered calcium ions could promote renal epithelial cell differentiation into cells with osteogenic phenotypes in idiopathic hypercalciuric patients (11). Typical bone osteoid proteins were also found to be significantly increased in hypercalciuric rats (12). These studies suggest that hypercalciuria may promote osteogenic-like differentiation of RPs.

MicroRNAs (miRNAs) are small noncoding RNA molecules of approximately $22 \mathrm{nt}$ in length. They have been shown to elicit post-transcriptional gene regulation by degradation or inhibition of target mRNA translation. In recent years, several studies have been reported that miRNAs are associated with bone formation and osteoporosis $(13,14)$. miRNA-410-3p is involved in a variety of biological processes and plays important roles in mammalian development. For instance, it has been implicated in the promotion of apoptosis in rhabdomyosarcoma cells and the attenuation of gemcitabine resistance in pancreatic ductal adenocarcinoma $(15,16)$. However, there are no reports that clarify the role of miRNA-410-3p in osteoblast differentiation.
Studies have shown that Msh homeobox 2 (MSX2) enhances osteogenic differentiation in synergy with bone morphogenetic-2 protein (BMP2). Furthermore, MSX2 was found to promote the expression of critical osteogenesis proteins [runt-related transcription factor 2 (Runx2) and Osterix] in vascular smooth muscle cells (17). However, little is known about the relationship between MSX2 and miRNA in the development of RP. We speculated that human renal interstitial fibroblasts (hRIFs), as one of the main cell components in renal papillary tissues, may have osteogenic-like differentiation potential, and osteogeniclike differentiation may occur under the influence of pathological factors such as high concentration of calcium ions. Thus, this study aimed to investigate whether calcium ions could promote the osteogenic-like differentiation of hRIFs and whether miRNA-410-3p could regulate MSX2 level in hRIFs. Our findings may provide novel concepts for kidney stone treatment.

\section{Methods}

\section{Tissue samples}

The study was conducted in accordance with the Declaration of Helsinki (as revised in 2013). The study was approved by the Ethics Committee of The Xiangya Hospital, Central South University (No. 201703258) and informed consent was taken from all the patients. RP tissues were obtained from patients with $\mathrm{CaOx}$ stones who underwent percutaneous nephrolithotomy. Donors of normal renal papillary (nRP) tissues were patients with renal tumors who underwent nephrectomy. Samples were obtained from papillary tissues with no tumor invasion. All samples were surgically collected and immediately stored at $-80{ }^{\circ} \mathrm{C}$.

\section{bRIFs isolation and culture}

hRIF isolation and culture proceeded as described by Rodemann et al. (18). hRIFs were obtained from normal human kidney papillary tissues $(10 \mathrm{~g})$. Cell isolation was performed with enzyme mix $[15 \mathrm{~mL}, 0.2 \%$ Collagenase I (Sigma-Aldrich, USA) and $0.25 \%$ trypsin (Sigma-Aldrich, USA) at $37{ }^{\circ} \mathrm{C}$ for $60 \mathrm{~min}$. The cell suspension was then filtered through a 200 -mesh nylon net and centrifuged at $800 \mathrm{rpm}$ for $5 \mathrm{~min}$. The culture medium (DMEM, 10\% fetal bovine serum (FBS; BI, Israel), $100 \mathrm{U} / \mathrm{mL}$ penicillin (BI, Israel) and $100 \mu \mathrm{g} / \mathrm{mL}$ streptomycin (BI, Israel)] was 
added to the precipitate, and the cells were maintained at $37{ }^{\circ} \mathrm{C}$ in $5 \% \mathrm{CO}_{2}$ for 1 hour, and then the culture medium was replaced with new media to filter the adherent fibroblasts. The culture medium was replaced every 3 days.

\section{$R N A$ extraction and quantitative real-time polymerase chain reaction ( $q P C R)$ analyses}

RNA extracted from tissue samples were isolated with Trizol reagent (Invitrogen, Carlsbad, CA, USA), according to the manufacturer's protocol. The primer sequences for qPCR are displayed in Table S1. qPCR analyses were performed on MX3000p real-time PCR (Agilent, USA). The relative miRNA and mRNA expression levels were analyzed using the $2^{-\Delta \Delta \mathrm{Ct}}$ method.

\section{Immunobistochemistry (IHC)}

IHC was performed to evaluate the osteogenesis-related proteins expression in RP tissue. Paraffin-embedded sections were dewaxed and rehydrated before treatment with $3 \%$ hydrogen peroxide for 30 minutes. Antigen retrieval and incubation with anti-Runx2 antibody (RRID: AB-10888180), anti-osteocalcin (anti-OCN) antibody, and anti-osteopontin (anti-OPN) antibody (RRID: AB2194998) were performed. After 24 h, the tissue sections were incubated with horseradish-conjugated secondary antibodies. Subsequently, all sections were stained with hematoxylin. Positive protein expression was indicated by brown granules in the cytoplasm or matrix. The staining intensity was graded into four levels: 0 was negative staining, 1 was low intensity, 2 was moderate intensity, and 3 was high intensity. The percentages of positively stained cells were also divided into four levels: 0 was $0 \%$, 1 was $\leq 25 \%, 2$ was $\leq 50 \%, 3$ was $\leq 75 \%$, and 4 was $\leq 100 \%$. Histological scores were determined by the sum of intensity scores and the percentage of positively stained cells. Protein expression levels were considered low if histological scores were $\leq 3$, while scores $\geq 4$ and $\leq 7$ were considered high.

\section{Osteogenic culture of hRIFs}

hRIFs were seeded in 6-well plates and cultured for $24 \mathrm{~h}$. Osteogenic medium containing 10\% FBS, 1\% L-glucose, $1 \%$ penicillin-streptomycin, $0.25 \mathrm{mM}$ ascorbic acid, $10 \mathrm{mM}$ b-glycerophosphate, and $10 \mathrm{nM}$ dexamethasone was applied to a hRIF group. Another hRIF group underwent osteogenic induction in medium containing $10 \mathrm{mmol} / \mathrm{L}$ calcium chloride dehydrate (Sigma, St. Louis, MO, USA). The control group was cultured with Dulbecco's Modified Eagle Medium/Nutrient Mixture F-12 (DMEM/ HAM'S-F12) and 10\% heat activated-FBS. The osteogenic and control media were both replaced every 3 days.

\section{3-(4,5-dimethylthiazol-2-yl)-2,5-diphenyltetrazolium bromide (MTT) assay}

The proliferation ability of hRIFs was evaluated by MTT assay according to the manufacturer's instructions (Roche Diagnostics GmbH, Mannheim, Germany). hRIFs cells were transferred into 96-well plates. MTT solution $(5 \mathrm{mg} / \mathrm{mL})$ was placed into every well and incubated for $4 \mathrm{~h}$. A microplate reader was used to examine the absorbance values at $490 \mathrm{~nm}$.

\section{Alizarin red staining}

Alizarin red staining was performed to estimate the deposits of calcium aggregates. After treatment with osteogenic induction medium, the hRIFs were washed twice with phosphate-buffered saline (PBS), fixed with $4 \%$ paraformaldehyde for $15 \mathrm{~min}$, and subsequently stained with $1 \%$ alizarin red staining for $5 \mathrm{~min}$. Calcified nodules were observed under an inverted microscope and the absorbance at $570 \mathrm{~nm}$ was read in triplicate.

\section{Western blot}

Western blot was conducted to examine the protein expression of target genes. After hRIFs were induced and transfected, cells were harvested for Western blot analysis. The cells were homogenized in ice-cold suspension and lysate (Beyotime, China) was added for total protein extraction. An equivalent amount of protein was fractionated on SDS polyacrylamide gels before immunoblotting with the following main antibodies: anti-Runx2 (Abcam, UK), anti-OCN (Abcam, UK) or anti-OPN (ZSGB-BIO, China). The membrane was incubated with a peroxidase-conjugated secondary antibody. The blotted bands were quantified using Image J (Bethesda, USA).

\section{Cell transfection}

hRIFs were transfected with miRNA-410-3p mimic, miRNA-410-3p inhibitor, MSX2 overexpression plasmid, or a negative control. Lipofectamine 2000 (Invitrogen, 

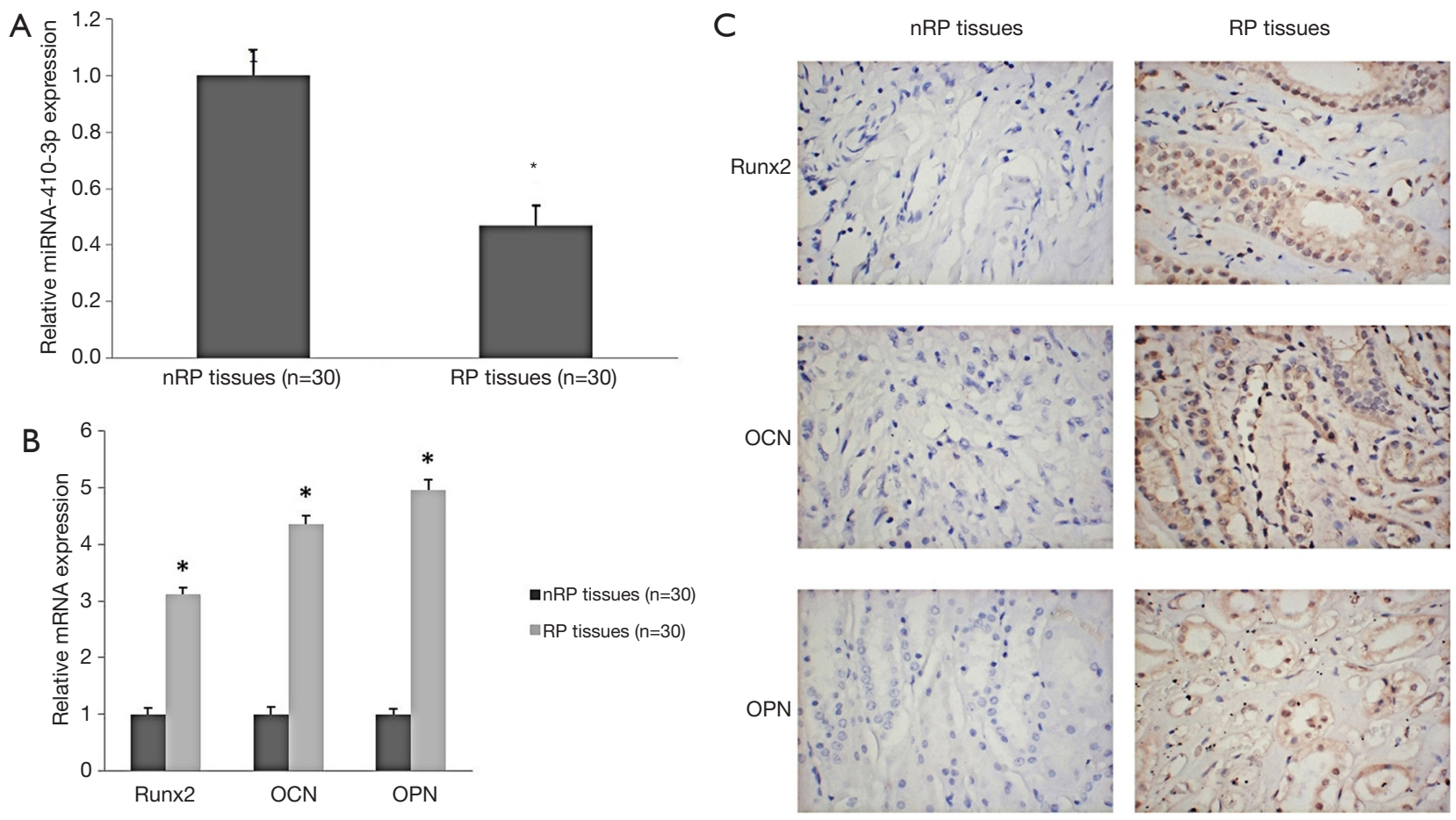

Figure 1 miRNA-410-3p was low expressed and osteogenesis related protein were high expressed in RPs tissues. (A) The level of miRNA410-3p was low in RP tissues; (B) qPCR showed the level of Runx2, OCN, OPN were higher in RP tissues; (C) the IHC showed Runx2, OCN, OPN were higher expressed in RP tissues $(\times 100)$. * $\mathrm{P}<0.05$. RP, Randall's plaque; Runx2, runt-related transcription factor 2; OCN, osteocalcin; OPN, osteopontin; IHC, immunohistochemistry; nRP, normal renal papillary.

USA) was used for transfection. The cells were harvested for subsequent experiments $48 \mathrm{~h}$ post-transfection.

\section{Dual-luciferase reporter gene assay}

TargetScan database version 7.1 (http://www.targetscan. org/vert_71/) was used to predict the target genes of miRNA-410-3p, and the wild-type or mutated MSX2 3'-UTR luciferase reporter vectors were designed. The vector was co-transfected with miRNA-410-3p mimics or miRNA-410-3p control into hRIFs using Lipofectamine 2000 (Invitrogen, USA). After culturing for $48 \mathrm{~h}$, luciferase activities were detected with the Dual Luciferase Reporter Gene Assay Kit (RG027; Beyotime).

\section{Statistical analysis}

Data are expressed as the mean \pm standard deviation (SD). The statistical significance between two groups was analyzed by a Student's $t$-test. P values $<0.05$ were considered statistically significant. Data analyses were performed using Statistical Package for the Social Sciences (SPSS version 17.0, Chicago, IL, USA).

\section{Results}

\section{Low expression of miRNA-410-3p in RP tissues}

qPCR analysis of miRNA-410-3p expression in RP and $n R P$ tissues displayed low levels of miRNA-410-3p in $\mathrm{RP}$ compared with control nRP tissues (Figure 1A). This suggests that miRNA-410-3p may participate in the progression of RP formation.

\section{Osteogenesis-related protein expression in RP tissues}

qPCR and IHC were employed to assess the expression levels of osteogenesis-related protein in RP and $\mathrm{nRP}$ tissues. qPCR revealed higher protein levels of Runx2, OCN, and OPN in RP tissues (Figure $1 B$ ), with the same trend noted via IHC staining (Figure $1 C$ ). The results indicate that osteogenic-like differentiation may participate in the 
A

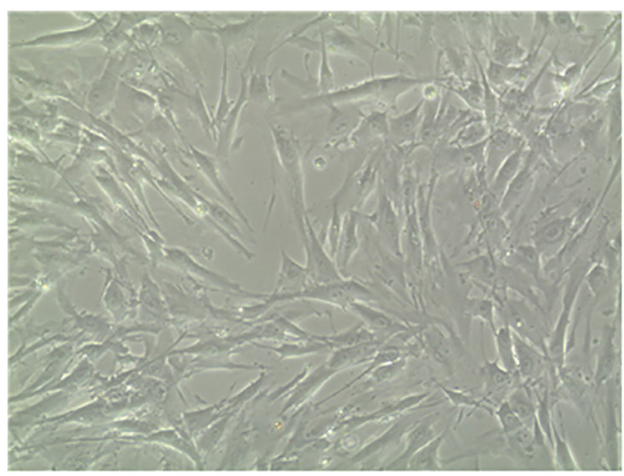

C

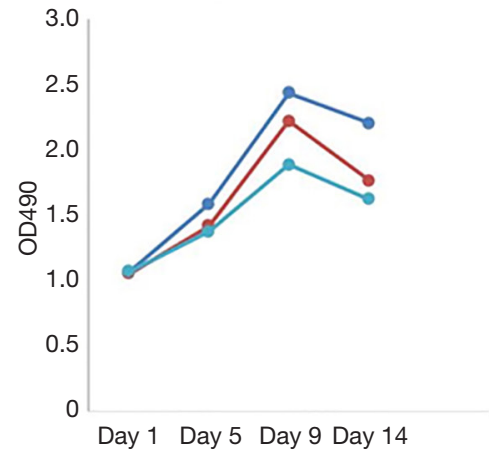

E

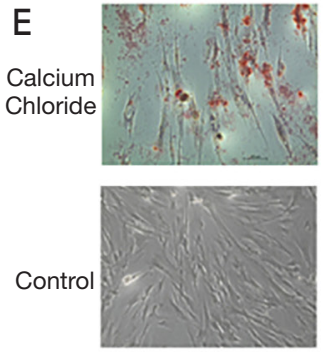

5 days
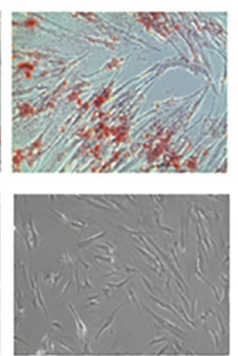

9 days
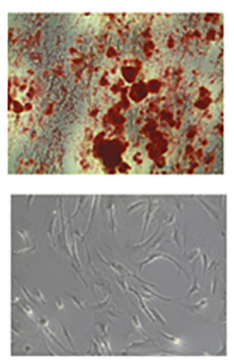

14 days
B

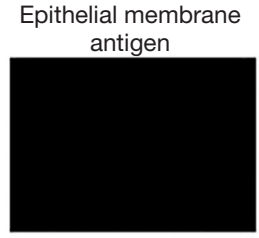

Vimentin
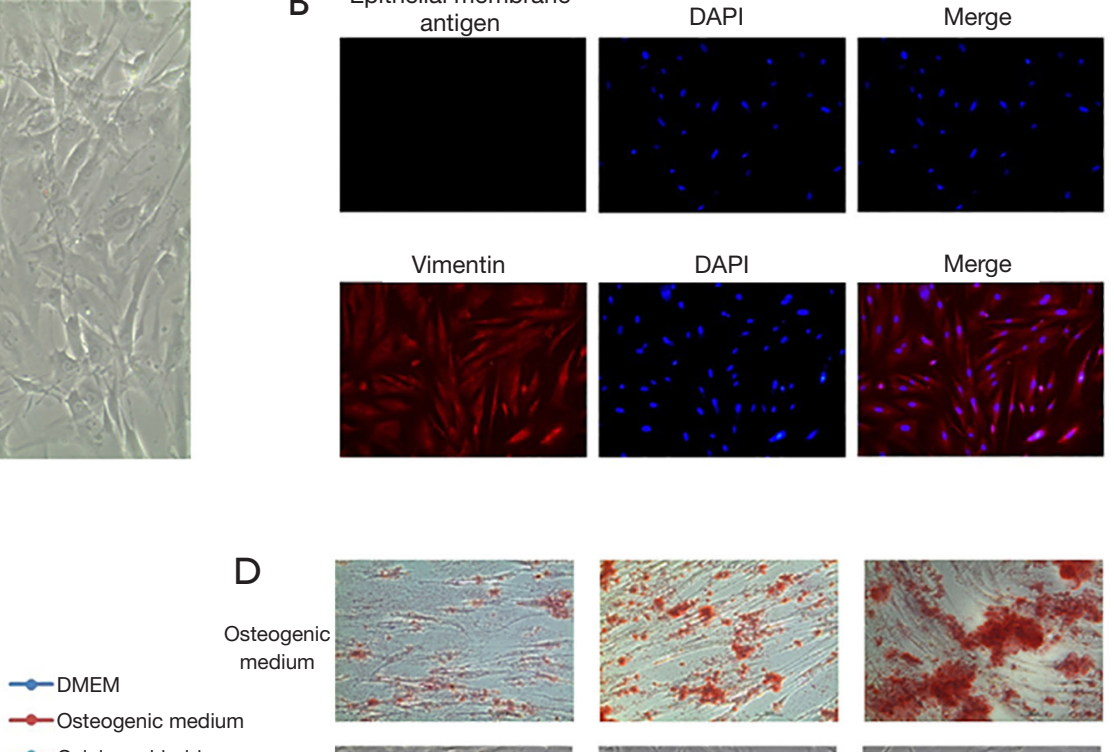

$\longrightarrow$ Calcium chloride

Control
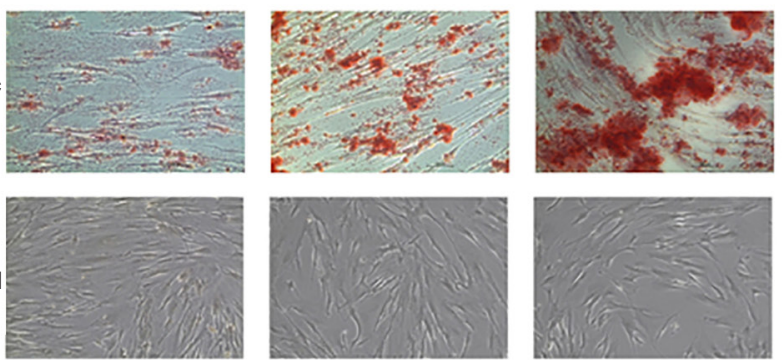

9 days

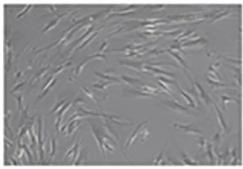

14 days
F

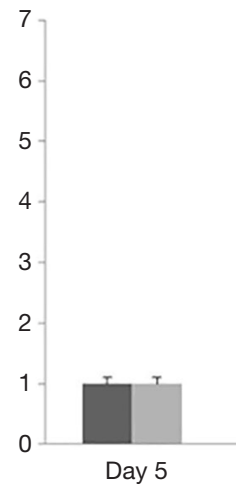

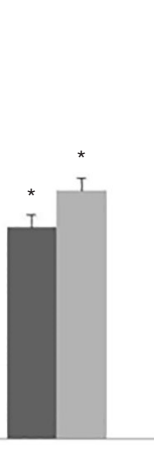

Day 9

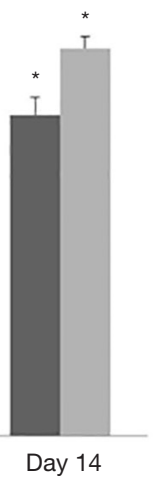

nOsteogenic medium

=Calcium chloride

Figure 2 The hRIFs possess osteogenic-like differentiation potential. (A) Primary cell culture showed hRIFs were shuttle-shaped cells ( $\times 100$ ); (B) immunofluorescence stain showed hRIFs were positive vimentin expression and negative epithelial membrane antigen expression ( $\times 100)$;

(C) MTT assay showed the cell proliferation ability of hRIFs was inhibited at 5, 9, 14 days compared to control group; (D) the hRIFs were treated with osteogenic medium, the calcium deposition showed a time-dependent trend and was most significantly at 14 days by alizarin red staining $(\times 100)$; $(\mathrm{E})$ the $\mathrm{hRIF}$ s were treated with calcium chloride, the calcium deposition showed a time-dependent trend and was most significantly at 14 days by alizarin red staining $(\times 100)$; (F) the absorbance was detected at $570 \mathrm{~nm}$, the results showed the deposits of calcium aggregates were significantly at 9 and 14 days by treated with osteogenic medium or calcium chloride. * $\mathrm{P}<0.05$. hRIFs, human renal interstitial fibroblasts; MTT assay, 3-(4,5-dimethylthiazol-2-yl)-2,5-diphenyltetrazolium bromide assay.

progression of RP formation.

\section{Establishment and identification of hRIFs}

hRIFs were isolated from human kidney papilla, and identified by morphological examination and immunofluorescence staining after extraction and culture. The results revealed hRIFs to be shuttle-shaped cells (Figure $2 A$ ) with positive vimentin expression and negative epithelial membrane antigen expression (Figure 2B). 

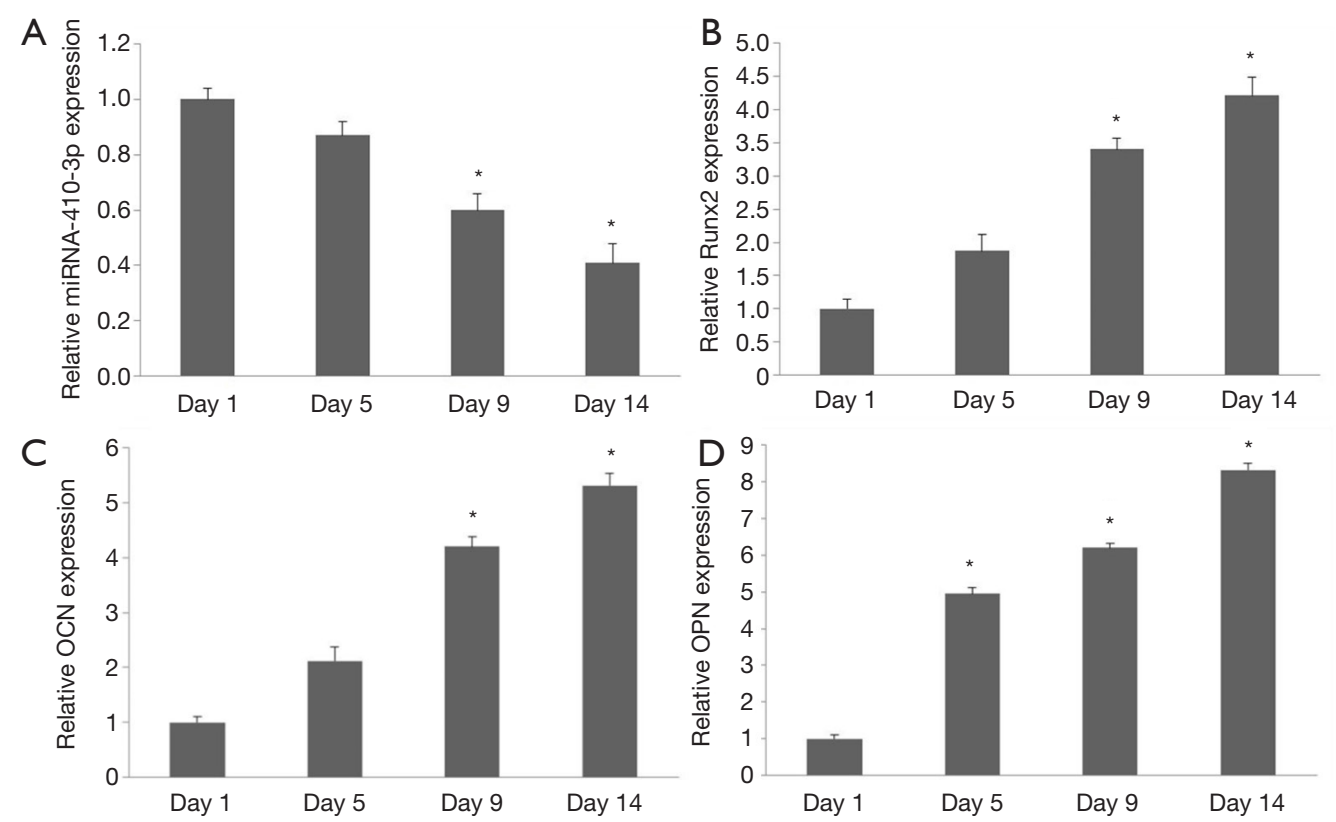

Figure 3 miRNA-410-3p and osteogenesis proteins showed a time-dependent trend during the hRIFs treated with calcium chloride. (A) miRNA-410-3p level gradually decreased with the prolongation of induction; (B,C,D) osteogenesis proteins Runx2, OCN and OPN level were increased with the prolongation of induction. * $\mathrm{P}<0.05$. hRIFs, human renal interstitial fibroblasts; Runx2, runt-related transcription factor 2; OCN, osteocalcin; OPN, osteopontin.

\section{The hRIFs showed osteogenic-like differentiation potential}

MTT assay was performed at 1, 5, 9, and 14 days after the hRIFs were treated with osteogenic medium or calcium chloride. The results showed that cell proliferation was inhibited at 5, 9, and 14 days compared to the control group (Figure 2C). Alizarin red staining was performed to estimate the deposits of calcium aggregates. Calcium deposition increased over time, and was most abundant at day 14 (Figure 2D,E,F). hRIFs treated with calcium chloride during osteogenic-like differentiation displayed decreasing miRNA-410-3p levels with the prolongation of induction (Figure $3 A$ ). In contrast, osteogenesis proteins Runx2 (Figure 3B), OCN (Figure 3C), and OPN (Figure 3D) levels increased under the same conditions. The results suggest that hRIFs possess osteogenic-like differentiation potential.

\section{Overexpression of miRNA-410-3p suppressed osteogenic- like differentiation of hRIFs}

Transfection of miRNA-410-3p mimics for $48 \mathrm{~h}$ significantly upregulated miRNA-410-3p level in hRIFs (Figure 4A). Alizarin red staining revealed decreased volumes of calcified nodules after overexpression of miRNA-410-3p in calcium chloride treated hRIFs (Figure 4B,C). Additionally, mRNA expression of Runx2 (Figure 4D), OCN (Figure 4E), and OPN (Figure $4 F$ ) also decreased after overexpression of miRNA-410-3p in calcium chloride treated hRIFs. These results indicate that miRNA-410-3p overexpression suppressed the osteogenic-like differentiation of hRIFs.

\section{Knock down miRNA-410-3p promoted osteogenic-like differentiation of hRIFs}

Transfection of miRNA-410-3p inhibitor for $48 \mathrm{~h}$ significantly downregulated miRNA-410-3p expression in hRIFs (Figure $5 A$ ). Alizarin red staining showed an increased volume of calcified nodules after miRNA-410-3p was downregulated in hRIFs treated with calcium chloride (Figure 5B,C). Meanwhile, the mRNA levels of Runx2 (Figure 5D), OCN (Figure 5E), and OPN (Figure 5F) were upregulated in the presence of knock down miRNA-410-3p. These results suggest that the downregulated miRNA-410$3 p$ promoted the osteogenic-like differentiation of hRIFs.

\section{miRNA-410-3p regulates the osteogenic-like} differentiation of hRIFs by inhibiting MSX2

To further confirm the inhibitory mechanism of 

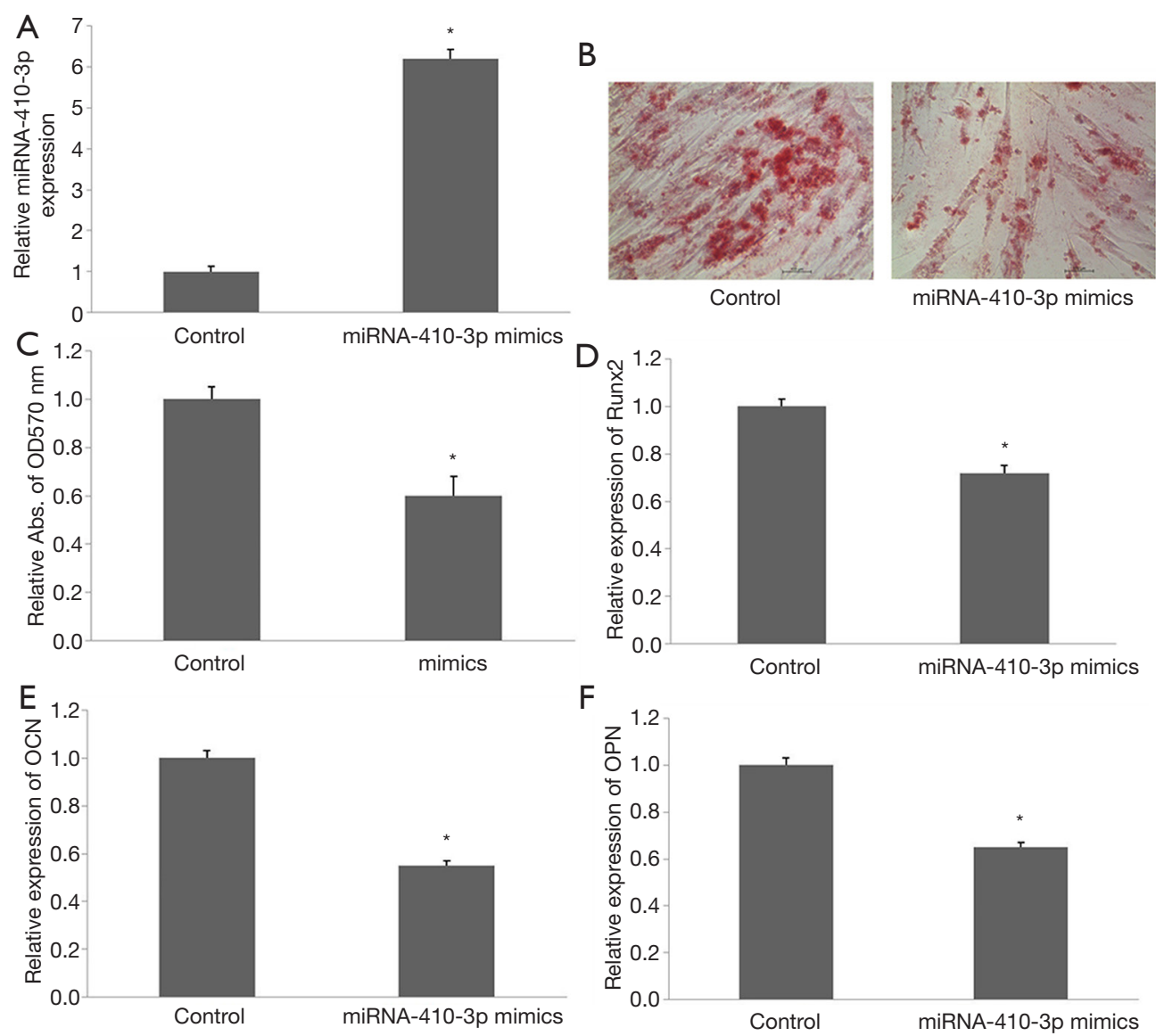

Figure 4 Overexpression of miRNA-410-3p suppressed osteogenic-like differentiation of hRIFs. (A) Transfection efficacy of miRNA-410$3 \mathrm{p}$ mimics in hRIFs for $48 \mathrm{~h}$; (B,C) alizarin red staining revealed the volume of calcification nodules were reduced after miRNA-410-3p overexpression in hRIFs treated with calcium chloride $(\times 100)$; (D,E,F) qPCR showed the mRNA levels of Runx2, OCN and OPN were downregulated after overexpression of miRNA-410-3p in hRIFs treated with calcium chloride. * $\mathrm{P}<0.05$. hRIFs, human renal interstitial fibroblasts; Runx2, runt-related transcription factor 2; OCN, osteocalcin; OPN, osteopontin.

miRNA-410-3p in hRIF osteogenic-like differentiation, bioinformatics analysis was performed. A potential binding sequence between miRNA-410-3p and MSX2 was identified by TargetScan (Figure $6 A$ ), with binding confirmed by the dual-luciferase reporter. Luciferase activity was reduced in cells co-transfected with miRNA-410-3p mimics and WT-MSX2 (Figure 6B). Furthermore, real-time qPCR and Western blot analysis revealed the ability of miRNA410-3p to effectively reduce the expression levels of MSX2 (Figure 6C,D), while the miRNA-410-3p inhibitor elevated MSX2 expression (Figure 6C,E). The above results suggested that miR-410-3p participated in the osteogeniclike differentiation of hRIFs by directly targeting MSX2.

\section{MSX2 promoted osteogenic-like differentiation of hRIFs}

To determine the effect of MSX2 on osteogenic-like differentiation in hRIFs, these cells were transfected with NC (non-specific control), miRNA-410-3p mimics, or miRNA-410-3p mimics plus MSX2 plasmids. MSX2 protein decreased when miRNA-410-3p was overexpressed. This phenomenon was reversed by MSX2 overexpression (Figure $7 A, B$ ). A negative correlation was observed between the volume and content of calcified nodules with miRNA410-3p overexpression. However, co-transfection of pcDNA-MSX2 (Figure 7C,D) led to a reversal of this trend. MSX2 was also shown to overcome the downregulation of 


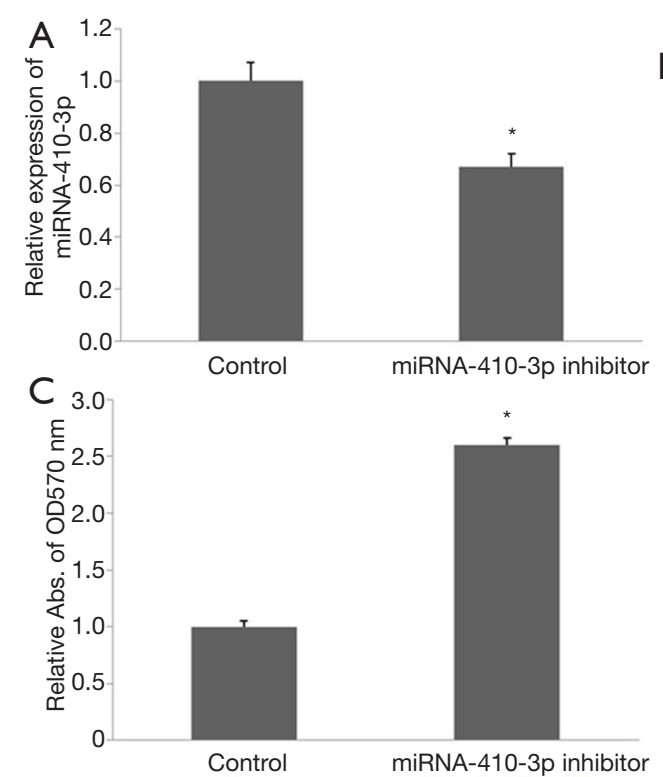

B
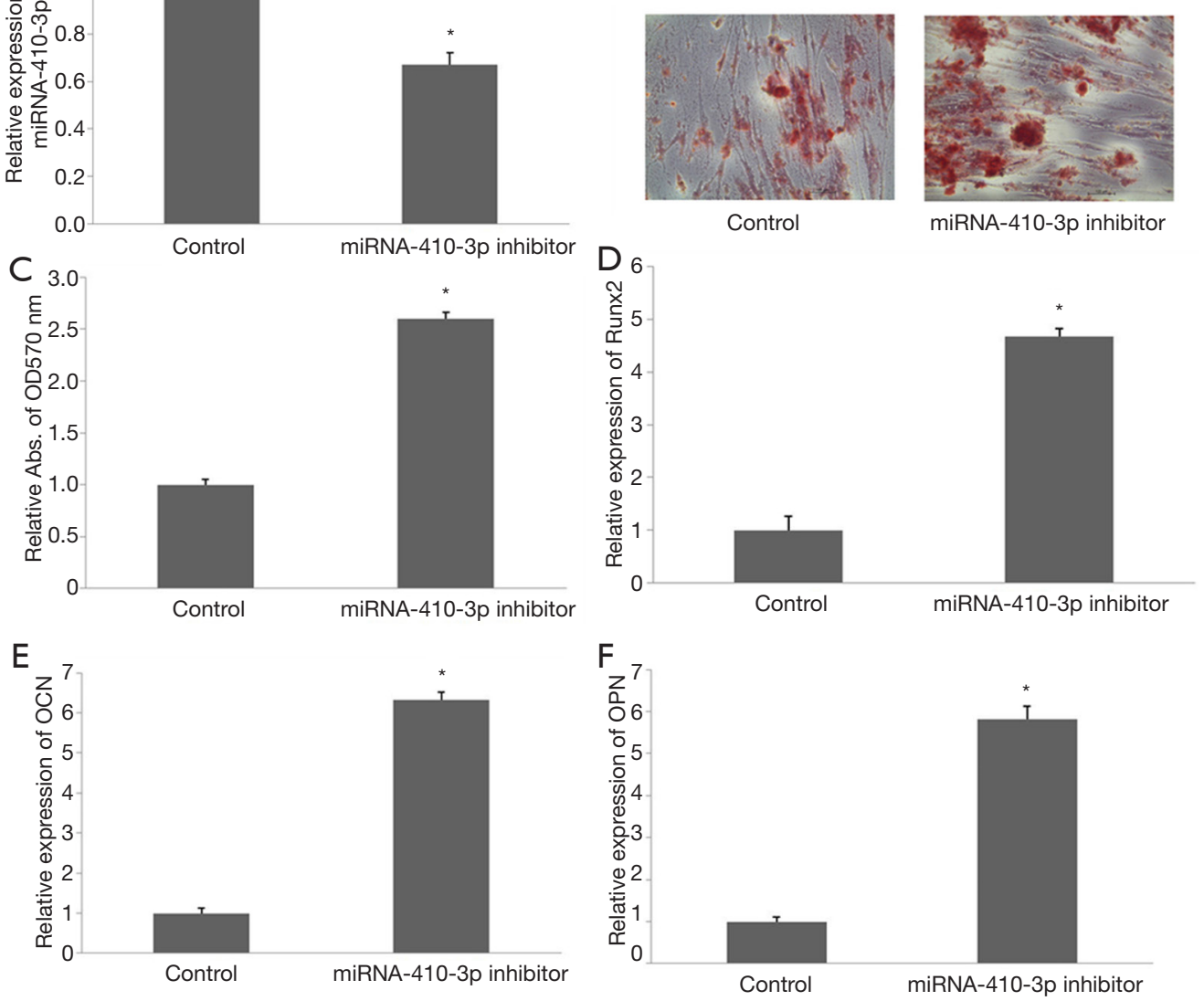

Figure 5 Knock down miRNA-410-3p promoted osteogenic-like differentiation of hRIFs. (A) Transfection efficacy of miRNA-410$3 \mathrm{p}$ inhibitor in hRIFs for $48 \mathrm{~h}$; $(\mathrm{B}, \mathrm{C})$ alizarin red staining revealed the volume of calcification nodules were increased after miRNA-4103 p downregulated in hRIFs treated with calcium chloride $(\times 100)$; (D,E,F) qPCR showed the mRNA levels of Runx2, OCN and OPN were upregulated after knock down miRNA-410-3p in hRIFs. *, P<0.05. hRIFs, human renal interstitial fibroblasts; qPCR, quantitative polymerase chain reaction; Runx2, runt-related transcription factor 2; OCN, osteocalcin; OPN, osteopontin.

Runx2 (Figure $7 E$ ) and Osterix (Figure $7 F$ ) during miRNA410-3p overexpression. The above findings demonstrate that low expression levels of miRNA-410-3p enhanced the upregulation effects of MSX2 on the osteogenic-like differentiation of hRIFs.

\section{Discussion}

In 1937, RP were identified and defined as interstitial deposits of calcium phosphate $(\mathrm{CaP})$ and calcium carbonate, appearing as "type I" papillary calcification on the renal papillary surface. These findings were based on the dissection of over 1,000 kidney pairs (19). In general, stone formation results from precipitation of urinary salts after exposure of primary plaques to urine $(20,21)$. RP, while observed in both non-stone formers and stone formers, is more commonly observed in the latter group (22-24). The presences of calcified lesions have been shown to be precursors of stone formation.

Analysis by Evan et al. clarified the microscopic structure of RP; they found that plaque originated in the basement membrane of the thin loops of Henle and extended through the interstitium to the region inferior to the urothelium (25). Khan et al., under scanning and transmission electron microscopic analysis (24), found plaque to appear as a small protuberance. Detailed scanning analyses showed plaques to be aggregates of spherical $\mathrm{CaP}$ crystals mixed with fibers of varying thickness and other cellular products. Urine 

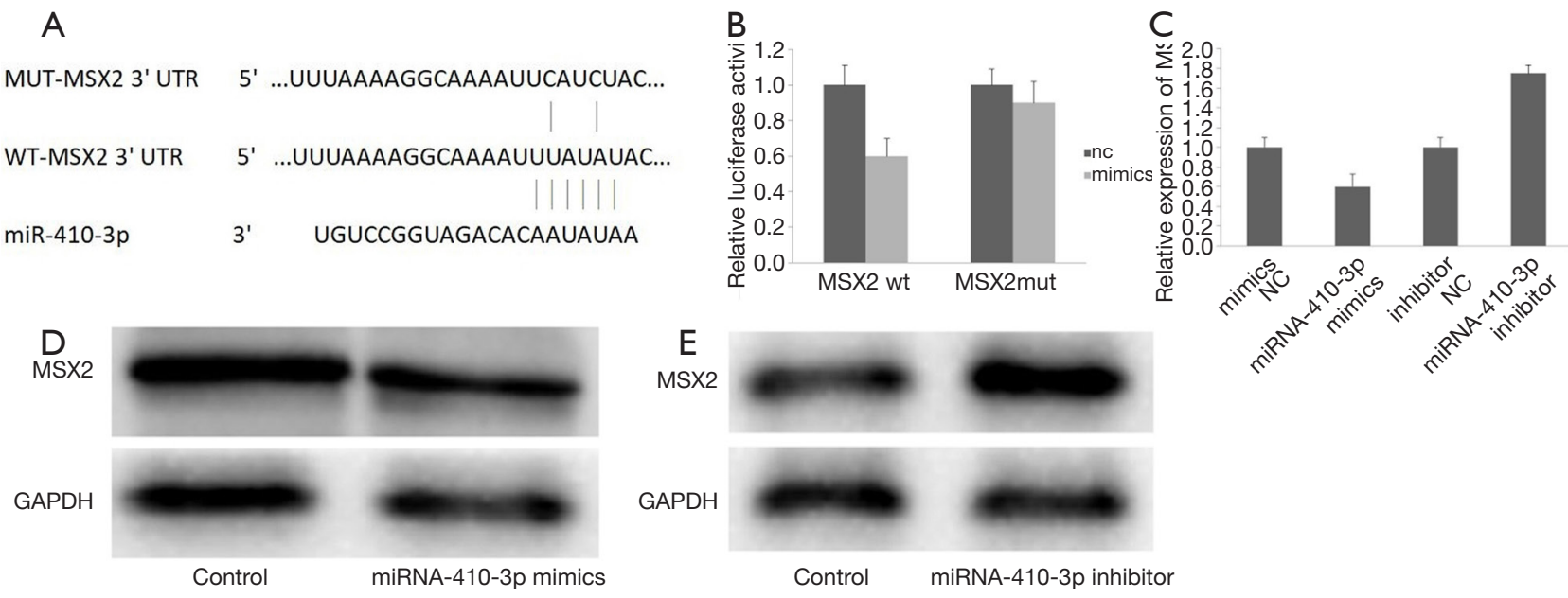

Figure 6 miRNA-410-3p regulates the osteogenic-like differentiation of hRIFs by inhibiting MSX2. (A) Potential binding sequences between miRNA-410-3p and MSX2; (B) luciferase activity in cells co-transfected with wt-MSX2/mut-MSX2 and miRNA-410-3p mimics; (C,D,E) qPCR and Western blot analysis documented that miRNA-410-3p mimics effectively reduced the expression levels of MSX2, while the miRNA-410-3p inhibitor elevated the expression of MSX2. hRIFs, human renal interstitial fibroblasts; MSX2, Msh homeobox 2; qPCR, quantitative polymerase chain reaction.

volume and calcium ion level were the main factors of plaque coverage (6). Collectively, these studies suggest that hypercalciuria is closely related to the formation of RP and kidney stones.

Ectopic calcification is an established process in various pathological processes, including kidney stone formation and atherosclerosis (26). There is a body of evidence which supports vascular calcification as an actively regulated process which is similar to bone development. Lineage tracing studies in mouse models have shown that vascular smooth muscle cells can transdifferentiate into osteochondrogenic cells (27). Bone osteoid proteins such as BMPs, Runx2, and MSX2 have been shown to be involved in these processes. Recent studies have found that renal epithelial cells also have the capacity to become osteogenic. Jia $e t$ al. have shown that bone-related factors (BMP2, Runx2, Osterix, and OPN) were significantly increased in genetic hypercalciuric rats (28). Using genome-wide analysis, Okada et al. found that the expression of OPN in renal tubular cells and the migration of macrophages in the interstitial space around crystals were necessary for stone formation (29).

Kageyama et al. found Madin-Darby canine kidney cells could produce $\mathrm{CaP}$ crystal on the basal side when grown in monolayers (30). When these cells were exposed to a highoxalate environment, and to $\mathrm{CaOx}$ and $\mathrm{CaP}$ crystals, the resulting NADPH oxidase activation and reactive oxygen production led to osteogenic phenotypes. Umekawa et $a l$. found oxalate and $\mathrm{CaOx}$ crystals upregulated OPN in renal fibroblasts (31). Evan et al. found that Runx2 was expressed in the RP of hypercalciuria patients (positive in the interstitial cells but not tubular epithelial cells) (32). However, idiopathic calcium stone formers showed no expression of Runx 2 at sites of RP. In the study of Mezzabotta et al., mesenchymal cells which did not produce calcified nodules stained positive for Runx2 and OPN (33). These studies indicated that the RP may be a perivascular niche in which epithelial and interstitial cells undergo osteogenic differentiation under specific conditions.

hRIFs are one of the main cells in kidney interstitial tissue. Studies have shown that the fibroblasts in multiorganization have osteogenic differentiation potential $(34,35)$. However, there are no reports on the osteogeniclike differentiation of hRIFs. Based on the above evidence, we speculated that hypercalciuria may induce the formation of RP by stimulating osteogenic-like differentiation of kidney interstitial tissue. In this study, we found osteogenesis-related proteins to be highly expressed in RP tissues. The hRIFs showed osteogeniclike differentiation potential after treatment with calcium. Additional investigations confirmed miR-410-3p regulated the osteogenic-like differentiation by directly targeting MSX2 in hRIFs. The low-expression of miRNA-410-3p led to a stimulatory effect of MSX2 on the osteogenic- 

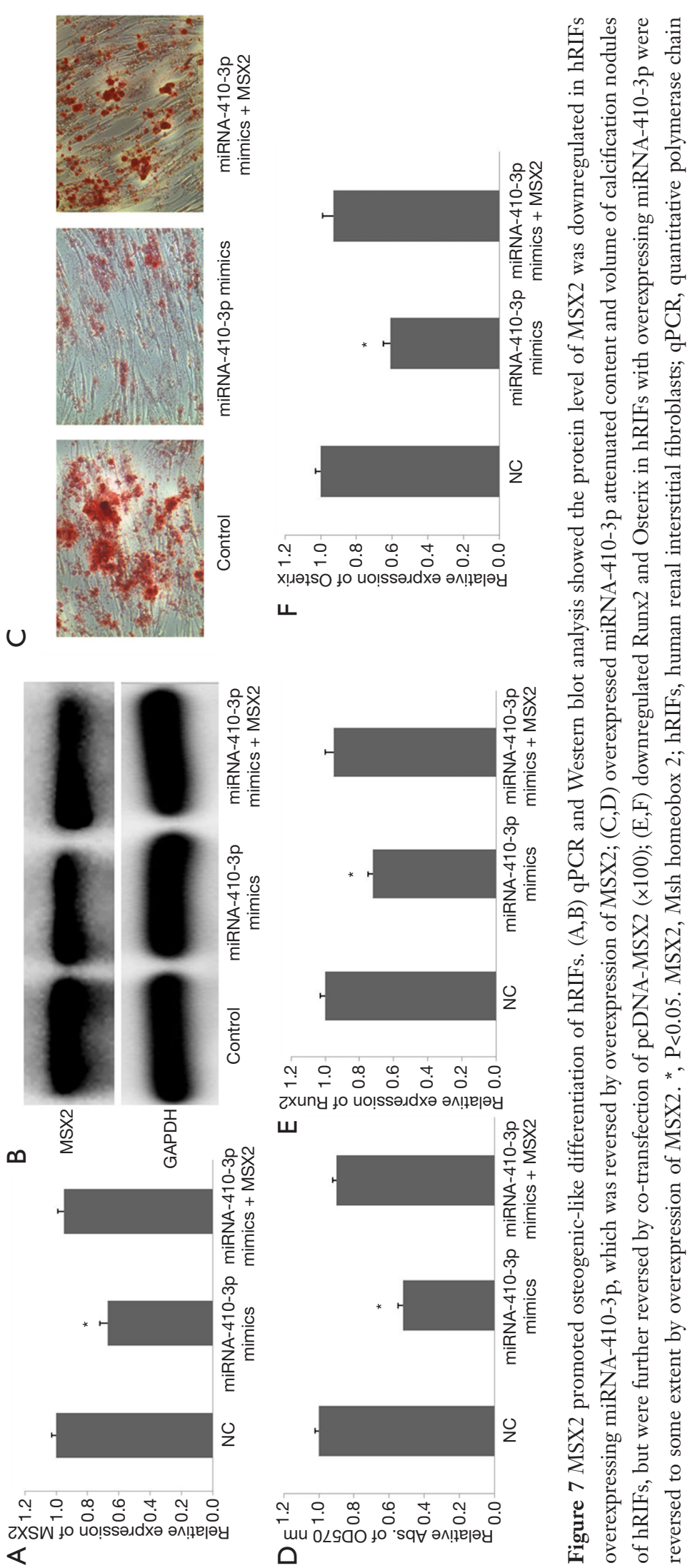

+ 芩当

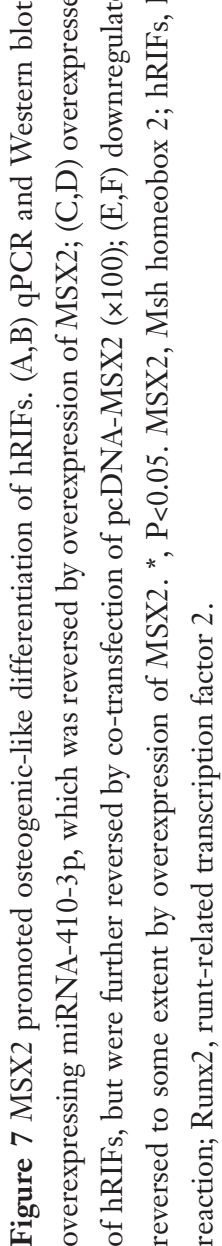


like differentiation of hRIFs via Runx2-Osterix signaling pathway.

In summary, the results of our study revealed that hRIFs have osteogenic-like differentiation potential, and that miRNA-410-3p regulates hRIFs osteogenic differentiation by inhibiting MSX2. These results are relevant to enhancing our understanding of the pathogenesis of kidney stones and indicate a novel potential therapeutic target.

\section{Acknowledgments}

Funding: This research was supported by grants from the National Natural Science Foundation of China (81770705) and the Youth Science Foundation of Xiangya Hospital (2019Q02).

\section{Footnote}

Data Sharing Statement: Available at http://dx.doi. org/10.21037/tau-20-607

Conflicts of Interest: All authors have completed the ICMJE uniform disclosure form (available at http://dx.doi. org/10.21037/tau-20-607). XZ serves as an unpaid editorial board member of Translational Andrology and Urology from Mar 2019 to Feb 2021. The other authors have no conflicts of interest to declare.

Ethical Statement: The authors are accountable for all aspects of the work in ensuring that questions related to the accuracy or integrity of any part of the work are appropriately investigated and resolved. The study was conducted in accordance with the Declaration of Helsinki (as revised in 2013). The study was approved by the Ethics Committee of the Xiangya Hospital, Central South University (No. 201703258) and informed consent was taken from all the patients.

Open Access Statement: This is an Open Access article distributed in accordance with the Creative Commons Attribution-NonCommercial-NoDerivs 4.0 International License (CC BY-NC-ND 4.0), which permits the noncommercial replication and distribution of the article with the strict proviso that no changes or edits are made and the original work is properly cited (including links to both the formal publication through the relevant DOI and the license). See: https://creativecommons.org/ licenses/by-nc-nd/4.0/.

\section{References}

1. Nirumand MC, Hajialyani M, Rahimi R, et al. Dietary plants for the prevention and management of kidney stones: preclinical and clinical evidence and molecular mechanisms. Int J Mol Sci 2018;19:765.

2. Scales CD Jr, Smith AC, Hanley JM, et al. Prevalence of kidney stones in the United States. Eur Urol 2012;62:160-5.

3. Ye Z, Zeng G, Yang H, et al. Efficacy and safety of tamsulosin in medical expulsive therapy for distal ureteral stones with renal colic: a multicenter, randomized, doubleblind, placebo-controlled trial. Eur Urol 2018;73:385-91.

4. Ratkalkar VN, Kleinman JG. Mechanisms of stone formation. Clin Rev Bone Miner Metab 2011;9:187-97.

5. Evan AP. Physiopathology and etiology of stone formation in the kidney and the urinary tract. Pediatr Nephrol 2010;25:831-41.

6. Kuo RL, Lingeman JE, Evan AP, et al. Urine calcium and volume predict coverage of renal papilla by Randall's plaque. Kidney Int 2003;64:2150-4.

7. Gambaro G, D'Angelo A, Fabris A, et al. Crystals, Randall's plaques and renal stones: do bone and atherosclerosis teach us something? J Nephrol 2004;17:774-7.

8. Priante G, Ceol M, Gianesello L, et al. Human proximal tubular cells can form calcium phosphate deposits in osteogenic culture: role of cell death and osteoblast-like transdifferentiation. Cell Death Discovery 2019;5:57.

9. Joshi S, Clapp WL, Wang W, et al. Osteogenic changes in kidneys of hyperoxaluric rats. Biochim Biophys Acta 2015;1852:2000-12.

10. Vernon HJ, Osborne C, Tzortzaki EG, et al. Aprt/Opn double knockout mice: osteopontin is a modifier of kidney stone disease severity. Kidney Int 2005;68:938-47.

11. He D, Wang S, Jia Z, et al. Calcium ions promote primary renal epithelial cell differentiation into cells with bone-associated phenotypes via transforming growth factor- $\beta 1$-induced epithelial-mesenchymal transition in idiopathic hypercalciuria patients. Mol Med Rep 2015;11:2199-206.

12. Jia Z, Wang S, Tang J, et al. Does crystal deposition in genetic hypercalciuric rat kidney tissue share similarities with bone formation? Urology 2014;83:509.e7-14.

13. Costa V, Carina V, Raimondi L, et al. MiR-33a controls hMSCS osteoblast commitment modulating the Yap/ Taz expression through EGFR signaling regulation. Cells 2019;8:1495. 
14. Feng Y, Wan P, Yin L, et al. The inhibition of MicroRNA139-5p promoted osteoporosis of bone marrow-derived mesenchymal stem cell by targeting Wnt/beta-catenin signaling pathway by NOTCH1. J Microbiol Biotechnol 2020;30:448-58.

15. Zhang L, Pang Y, Cui X, et al. MicroRNA-410-3p upregulation suppresses proliferation, invasion and migration, and promotes apoptosis in rhabdomyosarcoma cells. Oncol Lett 2019;18:936-43.

16. Xiong J, Wang D, Wei A, et al. MicroRNA-410-3p attenuates gemcitabine resistance in pancreatic ductal adenocarcinoma by inhibiting HMGB1-mediated autophagy. Oncotarget 2017;8:107500-12.

17. Matsubara T, Kida K, Yamaguchi A, et al. BMP2 regulates Osterix through Msx2 and Runx2 during osteoblast differentiation. J Biol Chem 2008;283:29119-25.

18. Rodemann HP, Müller GA. Characterization of human renal fibroblasts in health and disease: II. In vitro growth, differentiation, and collagen synthesis of fibroblasts from kidneys with interstitial fibrosis. Am J Kidney Dis 1991;17:684-6.

19. Randall A. The origin and growth of renal calculi. Ann Surg 1937;105:1009-27.

20. Randall A. Papillary pathology as precursor of primary renal calculus. J Urol 1940;44:9.

21. Sethmann I, Wendt-Nordahl G, Knoll T, et al. Microstructures of Randall's plaques and their interfaces with calcium oxalate monohydrate kidney stones reflect underlying mineral precipitation mechanisms. Urolithiasis 2017;45:235-48.

22. Stoller ML, Low RK, Shami GS, et al. High resolution radiography of cadaveric kidneys: unraveling the mystery of Randall's plaque formation. J Urol 1996;156:1263-6.

23. Khan SR, Canales BK. Unified theory on the pathogenesis of Randall's plaques and plugs. Urolithiasis 2015;43 Suppl 1:109-23.

24. Khan SR, Rodriguez DE, Gower LB, et al. Association of Randall plaque with collagen fibers and membrane vesicles. J Urol 2012;187:1094-100.

25. Evan AP, Lingeman JE, Coe FL, et al. Randall's plaque of patients with nephrolithiasis begins in basement membranes of thin loops of Henle. J Clin Invest 2003;111:607-16.

26. Demer LL, Tintut Y. Inflammatory, metabolic, and genetic mechanisms of vascular calcification. Arterioscler Thromb Vasc Biol 2014;34:715-23.
27. Speer MY, Yang HY, Brabb T, et al. Smooth muscle cells give rise to osteochondrogenic precursors and chondrocytes in calcifying arteries. Circ Res 2009; 104:733-41.

28. Jia Z, Wang S, He D, et al. Role of calcium in the regulation of bone morphogenetic protein 2, runt-related transcription factor 2 and Osterix in primary renal tubular epithelial cells by the vitamin D receptor. Mol Med Rep 2015;12:2082-8.

29. Okada A, Yasui T, Hamamoto S et al. Genome-wide analysis of genes related to kidney stone formation and elimination in the calcium oxalate nephrolithiasis model mouse: detection of stone-preventive factors and involvement of macrophage activity. J Bone Miner Res 2009;24:908-24.

30. Kageyama S, Ohtawara Y, Fujita K, et al. Microlith formation in vitro by Madin Darby canine kidney (MDCK) cells. Int J Urol 1996;3:23-6.

31. Umekawa T, Iguchi M, Uemura H, et al. Oxalate ions and calcium oxalate crystal-induced up-regulation of osteopontin and monocyte chemoattractant protein-1 in renal fibroblasts. BJU Int 2006;98:656-60.

32. Evan AP, Worcester EM, Williams JC Jr, et al. Biopsy proven medullary sponge kidney: clinical findings, histopathology, and role of osteogenesis in stone and plaque formation. Anat Rec (Hoboken) 2015;298:865-77.

33. Mezzabotta F, Cristofaro R, Ceol M, et al. Spontaneous calcification process in primary renal cells from a medullary sponge kidney patient harbouring a GDNF mutation. J Cell Mol Med 2015;19:889-902.

34. Zhang Z, Li X, Zhao J, et al. Effect of autogenous growth factors released from platelet concentrates on the osteogenic differentiation of periodontal ligament fibroblasts: a comparative study. PeerJ 2019;7:e7984.

35. Pasanisi E, Ciavarella C, Valente S, et al. Differentiation and plasticity of human vascular wall mesenchymal stem cells, dermal fibroblasts and myofibroblasts: a critical comparison including ultrastructural evaluation of osteogenic potential. Ultrastruct Pathol 2019;43:261-72.

Cite this article as: Cui Y, Zeng F, Zhu Z, Huang F, Chen J, He C, Li Y, Chen Z, Yang Z, Zu X, Chen H. Suppression of osteogenic-like differentiation in human renal interstitial fibroblasts by miRNA-410-3p through MSX2. Transl Androl Urol 2020;9(5):2082-2093. doi:10.21037/tau-20-607 
Supplementary

Table S1 Primer sequences for qPCR

\begin{tabular}{lll}
\hline Target genes & Classification & Primer sequences \\
\hline Runx2 & Forward & AGAAGGCACAGACAGAAGCTTGA \\
& Reverse & AGGAATGCGCCCTAAATCACT \\
OPN & Forward & ACACTTTCACTCCAATCGTCC \\
& Reverse & TGCCCTTCCGTTGTTGTCC \\
OCN & Forward & TCGTGTGTCTTCTCCACAGC \\
& Reverse & TGGCCACTTACCCAAGGTAG \\
Osterix & Forward & ATGGCGTCCTCTCTGCTTG, \\
& Reverse & TGAAAGGTCAGCGTATGGCTT \\
MSX2 & Forward & AGACATATGAGCCCCACCAC \\
& Reverse & CAAGGCTAGAAGCTGGGATG \\
miRNA-410-3p & Forward & AGTTGTTCACCACCTTCTCCAC \\
& Reverse & TATCGTTGTACTCCAGTCCAAGTC \\
\hline
\end{tabular}

qPCR, quantitative polymerase chain reaction; Runx2, runt-related transcription factor 2; OPN, osteopontin; OCN, osteocalcin; MSX2, Msh homeobox 2. 